\title{
Caliber persistent artery in the anterior palate of an Egyptian patient with type-2 diabetes mellitus: a rare case report
}

\author{
Bacem A.E.O. Khalele \\ Department of Molecular Pathology, Cairo University, Giza, Egypt
}

\begin{abstract}
This paper reports a very rare case of isolated ulcerative lesion of the palate in a patient with type- 2 diabetes mellitus. The patient refused to run any radiological tests, other than the periapical radiograph. This rendered the diagnostic dilemma more challenging. Through histochemical and immunohistochemical stains, the case could be non-invasively diagnosed as caliberpersistent artery. All differential diagnoses were excluded based on concrete findings.
\end{abstract}

\section{Introduction}

Diabetes mellitus encompasses a group of genetically and clinically heterogeneous metabolic disorders characterized by hyperglycemia depletion of antioxidants, and alteration in lipid metabolism. Diabetes mellitus (type 2) characterizes an insulin resistance, which progresses into an insulin deficiency due to a secondary failure in the pancreatic $\beta$-cells. Given the pathophysiology of diabetes-related complications, diabetic patients develop a relatively higher vulnerability of the mucosa upon exposure to various ulcerogens, ischemia or reperfusion, stress, and nonsteroidal anti-inflammatory drugs..$^{1-4} \mathrm{~A}$ wide diversity of inflammatory - either fungal, viral or bacterial - and neoplastic palatal lesions may overlap complicating the diagnosis of unusual rep-

Correspondence: Bacem A.E.O. Khalele, Department of Molecular Pathology, Cairo University, Rd. Giza, Giza Governorate, Egypt.

E-mail: bacemottoman@gmail.com

Key words: Pathology; diabetes.

Acknowledgments: my acknowledgments are sent to Prof. Dr. Brad W. Neville (SC, USA) and Dr. Buttota (ASU, Egypt) for the diagnostic help and guidance.

Conflict of interest: the author declares no potential conflict of interest.

Received for publication: 11 August 2015.

Revision received: 2 June 2016.

Accepted for publication: 1 June 2016.

This work is licensed under a Creative Commons Attribution NonCommercial 4.0 License (CC BY-NC 4.0).

CCopyright B.A.E.O. Khalele, 2017

Licensee PAGEPress, Italy

Italian Journal of Medicine 2017; 11:61-63

doi:10.4081/itjm.2017.635 resentations. Accordingly, meticulous care should be rendered to avoid unnecessary procedures and improper treatments, especially in chronic diabetic cases.

\section{Case Report}

A 66-year-old female, non-smoker and non-alcoholic, has suffered a painful palatal lesion. The ulcerative palatal lesion had an insidious onset, but turned painful for two weeks without any response to placebo (antibiotics, non-steroidal anti-inflamamtory drugs, anti-fungal and topical gels) (Figure 1A). An incisional biopsy was cut for microscopic examination.

No odontogenic irritants could be blamed. History of any concomitant tumor was negative. No running diseases in the family except for non-insulin dependent diabetes mellitus. The differential diagnosis included caliber-persistent artery with or without vasculitis, mucormycosis, lymphoma, granulomatosis polyangiitis (aka Wegener's granulomatosis), and spindle cell carcinoma.

Serum antinuclear antibody and C-reactive protein tests were negative. Detection of antineutrophil cytoplasmic antibodies (ANCA) from indirect immunofluorescence on blood was also negative ruling out granulomatosis polyangiitis. The periapical radiograph showed no more than normal maxillary trabeculae, excluding the liability of lymphocytic malignancies (Figure 2).

Histologically, the structure of this peripheral palatal lesion revealed an arterial blood vessel wall, with dense collagen with two layers of spindle cells running in different directions, surrounded by inflammatory infiltrates. Because of the surface ulceration and acute inflammation, the ulceration was expected to be either a sign for vasculitis or inflammatory evidence. Neither granuloma formation nor malignant features could be detected (Figure 3).

To exclude any fungal involvement, periodic acid- 
Schiff (PAS) stain was used to identify hyphae, if present. No fungal traces could be seen. Immunohistologically, the lesion was strongly positive for smooth-muscle actin, which stained the arterial wall. Other immunohistological tests were run to exclude any malignancy, including S-100, CK7, CK18 and p53, which were negative. In 10 days, the palatal site healed up dramatically confirming the inflammatory nature of this lesion (Figure 1B).

\section{Discussion}

As regards chronic diabetic complications, bone marrow-derived hematopoietic cells were recently considered proinflammatory and infiltrative to tissues inducing microvascular complications in addition to the dysregulated biochemical pathways downstream of hyperglycemia. ${ }^{5}$

Miko et al. first described, in 1980, a developmental anomaly referred to by them as persistent caliber artery. The artery becomes superficial toward the midline, and the persistent size makes it palpable, usually few millimeters inferior to the vermilion border. Being idiopathic in nature and rare in incidence, predisposing factors included intense sun exposure, senile atrophy of tissue associated with vascular ectasia, secondary to ageing, and injury from long-term pipe smoking. ${ }^{67}$ Although caliber-persistent artery is neither totally plausible nor delineated, it fits the best
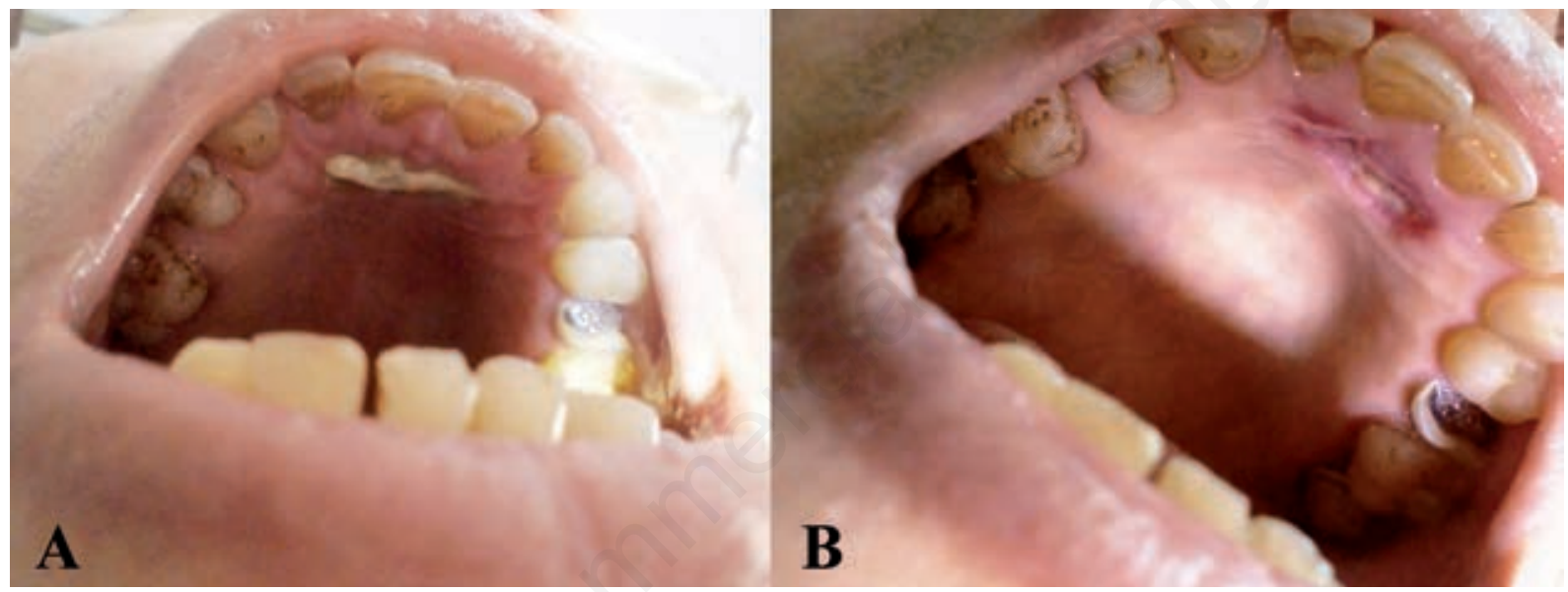

Figure 1. Clinical picture displaying ulcerated exophytic lesion, located at the anterior palate (A) before the biopsy and (B) 10 days after the biopsy.

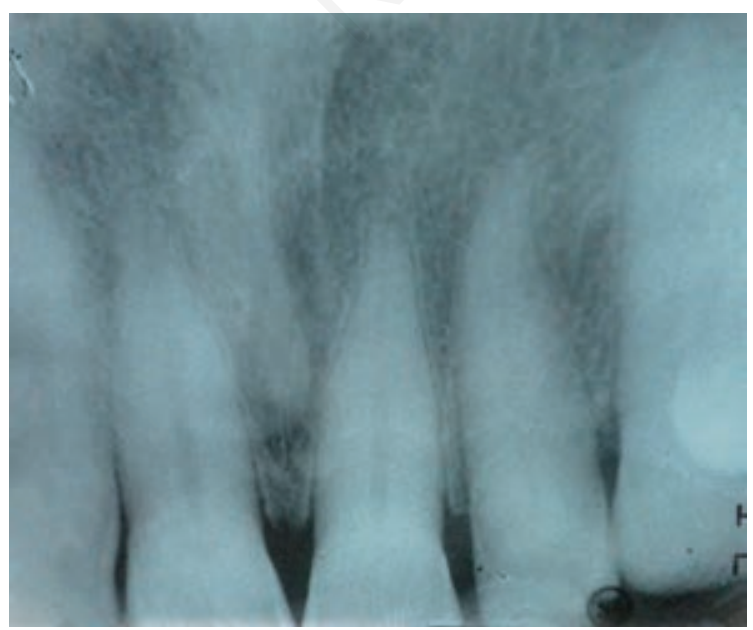

Figure 2. X-ray showing normal maxillary trabeculae. Neither dental caries nor any radicular pathosis is evident.

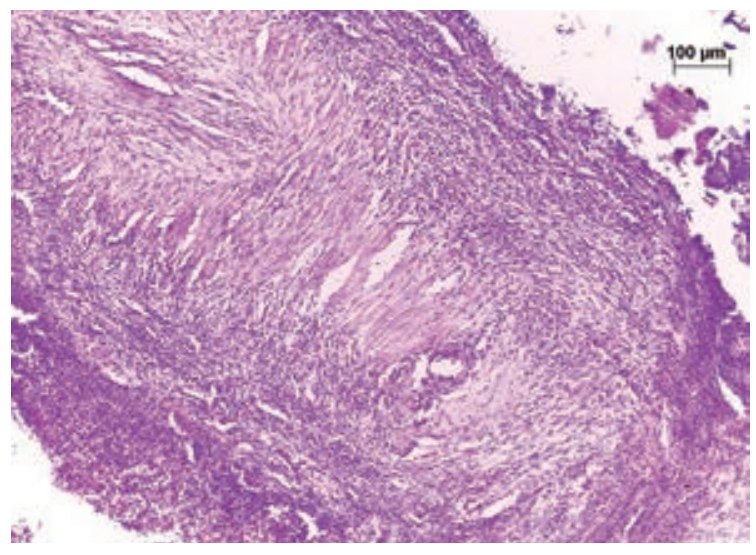

Figure 3. Photomicrograph revealing dense collagen of two layers of spindle cells running in different directions, surrounded by inflammation (H\&E stained, original magnification: $10 \times$ ). 
in this case. There appears a dysregulated hematopoietic element induced a low-grade irritation causing the arterial wall to thicken, which made the site liable to ulceration in the harsh oral environment. Either idiopathically induced or pathophysiologically caused by the underlying diabetic condition, the poorly vascularized anterior palate might contribute to the severity of the condition. Additionally, other possibilities of malignancies, infection, or autoimmunity were definitely ruled out.

For the deep fungal infections, which are not uncommon in diabetic patients, mucormycosis was frequently seen devastating the palate and encountering the nasal cavity. Mucormycosis is ubiquitous in nature and humans usually have a strong natural resistance to the infection. Mucormycosis becomes pathogenic when the patient's general resistance has been altered by metabolic disorders, immunosuppressive therapy, malignancy or other chronic debilitating disorders. An underlying disease, frequently diabetes mellitus, is always evident..$^{7-9}$ In this case, the recruitment of PAS stain, with its negative detection of hyphae, signed any deep fungal infection out.

Granulomatosis polyangiitis is an uncommon immune-based inflammatory necrotizing vasculitis with no certain etiology proven. Upper respiratory signs and symptoms include ear epistaxis, sinonasal dryness, crusts, obstruction, sinonasal destruction, subglottic stenosis, hearing loss, tinnitus and vertigo. Lower respiratory manifestations comprise parenchymal nodules, endobronchial lesions, pulmonary infiltrates, and pulmonary hemorrhage or embolism. Serologically, $90 \%$ of patients of granulomatosis polyangiitis are ANCA positive. ${ }^{10}$ None of the abovementioned signs and symptoms was evident in our case. Malignancies were histologically and immunohistologically excluded.

\section{Conclusions}

A better understanding of the versatile pathophysiology of diabetes-related complications is mandatory. The diagnostic procedures, especially in diabetic patients, should be careful and non-invasive. This should combine consultative efforts between medics and paramedics toward providing the best diabetic care for the patients.

\section{References}

1. Skyler J, Oddo C. Diabetes trends in the USA. Diabetes Metab Res Ver 2002;18:S21-6.

2. [No authors listed]. Report of the expert committee on the diagnosis and classification of diabetes mellitus. Diabetes Care 1997;20:1183-97.

3. Feingold K, Soued M, Serio M, et al. Multiple cytokines stimulate hepatic lipid synthesis in vivo. Endocrinology 1989;125:267-74.

4. Hink U, Li H, Mollnau H, et al. Mechanisms underlying endothelial dysfunction in diabetes mellitus. Circulation Res 2001;88:E14-22.

5. Kojima H, Kim J, Chan L. Emerging roles of hematopoietic cells in the pathobiology of diabetic complications. Trends Endocrinol Metab 2014;25:178-87.

6. Miko TL, Adler P, Endes P. Simulated cancer of the lower lip attributed to a "caliber persistent" artery. J Oral Pathol 1980;9:137-44.

7. Kua H, Blessing K, Holmes J, Burrell M. Calibre persistent artery of the lip: an underdiagnosed entity? J Clin Pathol 2000;53:885.

8. Manjunatha B, Das N, Sutariya R, Ahmed T. Mucormycosis of the hard palate masquerading as carcinoma Clin Pract 2012;2:e28.

9. Ruoppi P, Dietz A, Nikanne E, et al. Paranasal sinus mucormycosis: a report of two cases. Acta Otolaryngol 2001;121:948-52.

10. Ottoman B. Strawberry gingivitis of Wegener's granulomatosis: a clinico-pathological and immunohistochemical case study with review of literature. J Contemp Immunol 2015;2:59-66. 